\title{
Potentially Malignant Tools of Judgement: For Early Diagnosis
}

\section{Bintee Koirala ${ }^{1}$ and Karthik D Yadav ${ }^{2 *}$}

${ }^{1}$ Bachelor of Dental Surgery, India

${ }^{2}$ Master of Dental Surgery, Department of Oral Medicine and Radiology, India

*Corresponding Author: Karthik D Yadav, Master of Dental Surgery, Department of Oral Medicine and Radiology, India.
Received: March 10, 2020

Published: April 09, 2020

(C) All rights are reserved by Bintee Koirala and Karthik D Yadav.

\section{Abstract}

Oral cavity is often referred to as the reflection of the systemic condition of the body in health and disease. The most common oral mucosal diseases that have a very high malignant transformation rate are oral leukoplakia, oral erythroplakia, oral submucous fibrosis with the most frequently reported etiological agents being tobacco, alcohol, chewing of betel quid containing areca nut. Enormous research in the relevant field has reduced the time for reaching a conclusive diagnosis, but sometimes they are not as definite as a biopsy.

Keywords: Oral Cavity; Oral Leukoplakia; Oral Erythroplakia; Oral Submucous Fibrosis

\section{Introduction}

Oral cavity is often referred to as the reflection of the systemic condition of the body in health and disease. Some of the most common examples proving the same are the relation of diabetes and periodontal disease, the relation between hematopoietic system and gingival diseases and the relation between immune system with their signs and symptoms in the oral cavity.

The white lesions of the oral cavity forms a very big diagnostic challenge in the life of a general dentist, but neither are all lesions life threatening, nor are they lesions diagnosed correctly in the day to day practice. Most of these changes usually revert back to normal on its own without even recognition of it because of the magnanimous nature of the oral cavity and those who recognize it rarely get it treated at the earliest [1].

The progression of cancer formation is a two-stage course, with primarily the presence of a precursor/pre-cancer lesion and the advanced stage as a well-established one [2]. The term epithelial precursor lesions has been used In the WHO monograph on Head and Neck Tumors. The term potentially malignant disorders was recommended at a workshop coordinated by the WHO Collaborating Centre for Oral Cancer and Precancer in UK for the Precancerous lesions of the oral mucosa and was called so as it conveys that not all disorders described under this term may transform into cancer $[2,3]$.

The most common oral mucosal diseases that have a very high malignant transformation rate are oral leukoplakia, oral erythroplakia oral submucous fibrosis with the most frequently reported etiological agents being tobacco, alcohol, chewing of betel quid containing areca nut. A quick and prompt diagnosis can be lifesaving; hence its importance cannot be ignored as in later stages they might progress to severe dysplasia and even carcinoma in situ and/or squamous cell carcinoma [2-5].
Screening and diagnostic aids of oral potentially malignant disorders [3-5]:

- Standard screening test
1. Conventional oral examination

- Established diagnostic adjuncts

1. Toluidine Blue

2. Oral cytology

- Recently available light detection systems

1. MicroLux DL

2. ViziLite, ViziLite Plus

3. VELscope

4. Colposcopy

Conventional oral examination

Conventional oral examination has shortcomings which embrace false positive findings, making other diagnostic modalities the need of the hour. White lesions sometimes diagnosed falsely as leukoplakia is a catastrophe for the patient as it is a potentially malignant lesion [6]. Even though it is a premalignant change, the chances of it getting converted into cancer is rare $[6,7]$.

\section{Vital tissue staining}

Tolonium chloride (Toluidine blue) is used as a diagnostic stain as it stains mitochondrial DNA, dysplastic cells with increased DNA content or modified DNA in carcinogenic cells [7]. The application of toluidine blue which is a metachromatic dye recognizes malignant alterations and potential areas of high-grade dysplasia.

\section{Exfoliative cytology}

OralCDx brush biopsy is an oral transepithelial biopsy system that uses computer-assisted brushing. It is the study and interpretation of the characteristics of cells that wither off, naturally or artificially from the oral mucosa $[8,9]$. 
They are of significance in monitoring several sites for a large lesion and can also guide the choice of sites for incisional biopsies. It was designed to screen innocuous looking oral epithelial abnormalities for dysplasia or cancer $[8,9]$.

The detection of abnormal cells by this technique has a sensitivity of $52-100 \%$ and specificity $29-100 \%$. Overall, the OralCDx technique appears to overestimate dysplastic lesions and produce a high number of false-positive results [8,9].

\section{Vizilite plus}

ViziLite Plus employs a disposable chemiluminescent light packet with a wavelength in the range of 490 to $510 \mathrm{~nm}$. Chemiluminescence takes place when a chemical reaction produces light with varying intensity, the period and color depends on the type of reaction $[8,9]$.

Manufacturers claim that these devices enhance tissue reflectance of oral tissues after rinsing the mouth with $1 \%$ acetic acid for a minute. The hypothesis is that a cytoplasmic dehydration agent such as acetic acid alters the refractive properties of oral mucosal abnormalities with increased nuclear: cytoplasmic ratio [8,9].

Thus, dysplastic lesions become more visible as they appear white against the light blue appearance of the normal tissue, hence termed "acetowhite lesion" [8].

\section{Microlux DL}

Microlux/DL utilizes acetic acid followed by the use of diffused blue-white light-emitting diode with an fiber-optic light used to guide the low-energy blue light. The light emitting diode (LED) transilluminator is an autoclavable light guide that produces diffused light. After rinsing with the acetic acid solution, the manufacturer claim that abnormal cells will take on a whitish hue in contrast to the surrounding tissues making it more evident to the examiner $[8,10]$.

\section{VELscope}

Acronym for Visually Enhancing Lesion Scope (VELscope). This device utilizes blue light 400 to $460 \mathrm{~nm}$ in wavelength to cause tissue autofluorescence, which utilizes the fact that cellular fluorophores are excited by high intensity light of particular wavelengths $[8,10]$.

In a subject with healthy oral epithelium when viewed directly viewed through a narrow-band filter, as green-red fluorescence it appears pale green. This is because of excited fluorophores in the oral tissues. However, in dysplastic cells or potentially early tumors it appears dark green to black due to the cellular and structural changes which occur in neoplastic tissue. It is based on the fact that both the superficial epithelium and underlying stroma of developing premalignant lesions have changes in their optical properties $[8,10]$.

Cellular alterations such as decreased flavin adenine dinucleotide concentration and collagen matrix breakdown have been associated with loss of fluorescence visualization (FV). Furthermore, decreased fluorescence as a result of increased absorption and scattering of light occurs when there are structural changes in both the epithelium and lamina propria such as hypertrophy, hyperchromatism, cellular/nuclear pleomorphism and increased microvascularity [8].

\section{Colposcope}

Colposcope is an instrument used to identify visible clues suggestive of abnormal tissue. It was invented by Professor Hinselmann of Hamburg, Germany, in 1925 specifically for the purpose of detecting early cervical cancer [11].

Colposcopy is a commonly used technique in the study of cervical morphology using stereoscopic binocular magnification provided by the colposcope.

Colposcopy is a diagnostic method useful for the diagnosis and evaluation of cervical intra epithelial neoplasia and preclinical invasive cancer which permits magnified visualization of the site where cervical carcinogenesis occurs [11,12].

It helps in the process of directed biopsy and also in delineating the extent of lesions on the cervix in screen-positive women. In adjunct it also helps in directing treatments such as cryotherapy and loop electrosurgical excision procedure for cervical intraepithelial neoplasia $[11,12]$.

In a study done to evaluate the reliability of toluidine blue staining and colposcopy in determining biopsy site among patients with oral leukoplakia by Karunakar., et al. showed that the distribution of histopathologic diagnosis by Colposcopic and Toluidine blue examination in which 5(20\%) showed hyperkeratosis, 16 (64\%) cases showed mild dysplasia, moderate epithelial dysplasia was seen in $4(16 \%)$ of the cases $[11,12]$.

Out of 20 cases of homogenous leukoplakia, 14 were diagnosed with $76 \%$ accuracy for biopsy site in homogenous leukoplakia cases and all 5 cases of non-homogenous leukoplakia were correctly diagnosed with $100 \%$ accuracy. Out of 25 leukoplakia cases $19(76 \%)$ cases were accurately diagnosed for the biopsy site with both the Toluidine blue and Colposcopic $[11,12]$.

\section{Cell and tissue markers}

Epithelial growth factor (EGF), Cyclins, AgNOR, bcl2 and telomerase are the different tumor markers that have been evaluated [13]. Other tumor suppression markers and anti-tumor response like Retinoblastoma protein, p53 and Cyclin-dependent kinase inhibitors also are evaluated [13]. Angiogenic biomarkers CD105 and Eph receptor tyrosine kinases (Ephs), vascular EGF and four hypoxia biomarkers (GLUT-1, carbonic anhydrase IX, hypoxia inducible factor $1 \mathrm{a}$, and erythropoietin receptor) were also identified as biomarkers [14].

The matrix metallo-proteins are proteases typically expressed by invasive cancers and the contiguous stroma and their expression has often been reviewed in various studies [15]. 


\section{Positron emission tomography}

Fluoro-deoxyglucose positron emission tomography (FDG-PET) examination demonstrates precise and prognostic significance while defining lymphatic condition. It aids in timely assessment and diagnosis of oral malignancy in affected patients [16-30]. PET/ computed tomography (CT) can find and discriminate between the surgical and radiation-induced variations from residual or recurrent neoplasia because cancerous cells uphold greater FDG for lengthier intervals of time as compared to infectious and inflammatory structures [16-30].

\section{Bio-nanochip}

Recently, a novel bio-nanochip (BNC) sensor which is a fast oralcytology test that amalgamates the power of cytological morphometric examination with quantification of neoplastic biomarkers was documented [31]. Generally, microfluidics technology (labon-a chip) is the adjustment, miniaturization, amalgamation, and automation of analytical laboratory procedures into a solitary chip [32]. The conducted study on quantitative BNC method to oral cytology effectively revealed cancerous and precancerous conditions in a short time duration ( $<45$ minutes) [31].

The recognition of cancerous cells in the BNC sensor utilized membrane-related cell proteins that are especially present on the cellular membrane structure of neoplastic cells [33].

\section{Conclusion}

Over the years, millions have been spent to develop diagnostic techniques and equipment which are helpful to the treating diagnostician, all of which leads to the proper diagnosis and initiation of the treatment at an early stage, which means a good prognosis. Enormous research in the relevant field has reduced the time for reaching a conclusive diagnosis, but sometimes they are not as definite as a biopsy.

\section{Bibliography}

1. Burket LW., et al. "Burket's oral medicine”. PMPH-USA (1997): 124-156.

2. Shukla A. "Potentially Malignant Disorders of the Oral Cavity: A Clinical Study". Indian Journal of Otolaryngology and Head and Neck Surgery 66.1 (2013): 79-85.

3. Rai S., et al. "Future prospective and current status of antioxidants in premalignant and malignant lesions of oral cavity". International Journal of Nutrition, Pharmacology, Neurological Diseases 4.4 (2014): 198-202.

4. Masthan K., et al. "Leukoplakia: A short review on malignant potential". Journal of Pharmacy and Bioallied Sciences 7 (2015): S165-S166.

5. Gurkan Yardimci., et al. "Precancerous lesions of oral mucosa". World Journal of Clinical Cases 2.12 (2014): 866-872.

6. Lingen MW., et al. "Critical evaluation of diagnostic aids for the detection of oral cancer". Oral Oncology 44 (2008): 10-22.
7. Epstein JB., et al. "Advances in the diagnosis of oral premalignant and malignant lesions". Journal of the Canadian Dental Association 68.10 (2002): 617-621.

8. Epstein JB., et al. "Analysis of oral lesion biopsies identified and evaluated by visual examination, chemiluminescence and toluidine blue". Oral Oncology 44.6 (2008): 538-544.

9. Diz P., et al. "Oral leukoplakia and erythroplakia: A protocol for diagnosis and management". EAOM-Diagnostic and therapeutic protocols Oral leukoplakia and erythroplakia (2011).

10. Gray M., et al. "The clinical effectiveness of toluidine blue dye as an adjunct to oral cancer screening in general dental practice" (2000).

11. Pallagatti S., et al. "Colposcopy: a new ray in the diagnosis of oral lesions”. Indian Journal of Dental Research 22.6 (2011): 810-815.

12. Gynther GW., et al. "Direct oral microscopy and its value in diagnosing mucosal lesions: a pilot study". Oral Surgery, Oral Medicine, Oral Pathology, and Oral Radiology 90.2 (2000): 164167.

13. Chimenos-Küstner E., et al. "Oral cancer risk and molecular markers”. Medicina Oral, Patología Oral y Cirugía Bucal 9 (2004): 381-384.

14. Oliveira LR., et al. "Prognostic significance of immunohistochemical biomarkers in oral squamous cell carcinoma". International Journal of Oral and Maxillofacial Surgery 40.3 (2011): 298-307.

15. Lyons AJ and Jones J. "Cell adhesion molecules, the extracellular matrix and oral squamous carcinoma". International Journal of Oral and Maxillofacial Surgery 36.8 (2007): 671-679.

16. Masthan KM., et al. "Advanced diagnostic aids in oral cancer". Asian Pacific Journal of Cancer Prevention 13.8 (2012): 35733576.

17. Mittal N., et al. "Rose Bengal staining - diagnostic aid for potentially malignant and malignant disorders: a pilot study". Indian Journal of Dental Research 23.5 (2012): 561-564.

18. Du GF., et al. "Rose bengal staining in detection of oral precancerous and malignant lesions with colorimetric evaluation: a pilot study". International Journal of Cancer 120.9 (2007): 1958-1963.

19. Messadi DV. "Diagnostic aids for detection of oral precancerous conditions". International Journal of Oral Science 5.2 (2013): 59-65.

20. Kerr AR., et al. "Clinical evaluation of chemiluminescent lighting: an adjunct for oral mucosal examinations". The Journal of Clinical Dentistry 17.3 (2006): 59-63.

21. Farah CS., et al. "A pilot case control study on the efficacy of acetic acid wash and chemiluminescent illumination (ViziLite) in the visualisation of oral mucosal white lesions". Oral Oncology 43.8 (2007): 820-824. 
22. Shashidara R., et al. "Chemiluminescence: a diagnostic adjunct in oral precancer and cancer: a review". Journal of Cancer Research and Therapeutics 10.3 (2014): 487-491.

23. Trullenque-Eriksson A., et al. "Analysis of new diagnostic methods in suspicious lesions of the oral mucosa". Medicina Oral, Patología Oral y Cirugía Bucal 14.5 (2009): E210-E216.

24. Awan KH., et al. "Evaluation of an autofluorescence based imaging system (VELscop) in the detection of oral potentially malignant disorders and benign keratoses". Oral Oncology 47.4 (2011): 274-277.

25. Patton LL., et al. "Adjunctive techniques for oral cancer examination and lesion diagnosis: a systematic review of the literature". Journal of the American Dental Association 139.7 (2008): 896-905.

26. Shin D., et al. "Advances in fluorescence imaging techniques to detect oral cancer and its precursors". Future Oncology 6.7 (2010): 1143-1154.

27. Poh CF., et al. "Squamous cell carcinoma and precursor lesions: diagnosis and screening in a technical era". Periodontology 57.1 (2011): 73-88.

28. Olivo M., et al. "Advances in bio-optical imaging for the diagnosis of early oral cancer". Pharmaceutics 3.3 (2011): 354-378.

29. Roblyer D., et al. "Objective detection and delineation of oral neoplasia using autofluorescence imaging". Cancer Prevention Research 2.5 (2009): 423-431.

30. Onizawa K., et al. "Fluorescence photography as a diagnostic method for oral cancer". Cancer Letter 108.1 (1996): 61-66.

31. McDevitt J., et al. "A new bio-nanochip sensor aids oral cancer detection". SPIE Newsroom (2011).

32. Mehrotra R and Gupta DK. "Exciting new advances in oral cancer diagnosis: avenues to early detection". Head and Neck Oncology 3 (2011): 33.

33. Ziober BL., et al. "Lab-on-a-chip for oral cancer screening and diagnosis". Head Neck 30.1 (2008): 111-121.

\section{Assets from publication with us}

- Prompt Acknowledgement after receiving the article

- Thorough Double blinded peer review

- Rapid Publication

- Issue of Publication Certificate

- High visibility of your Published work

Website: https://www.actascientific.com/

Submit Article: https://www.actascientific.com/submission.php Email us: editor@actascientific.com

Contact us: +919182824667 